\title{
Assessment of modified Forssell's myectomy success rate in the treatment of crib biting in horses
}

\author{
Šárka Krisová, Zdeněk Žert, Kristína Žuffová \\ University of Veterinary and Pharmaceutical Sciences, Faculty of Veterinary Medicine, Equine Clinic, \\ Department of Surgery and Orthopaedics, Brno, Czech Republic
}

Received June 6, 2014

Accepted November 26, 2014

\begin{abstract}
The objective of this retrospective study was to assess the success rate of Forssell's modified myectomy - surgical treatment of crib biting on 33 horses of various breeds identified as stereotypical cribbers which were referred to the Equine Clinic in Brno between January 2001 and December 2010. The long term outcome was available for all horses. The overall success of the surgery without considering influencing factors was $61 \%$. Thirteen horses $(39 \%)$ returned to the stereotypical behaviour sooner or later after the surgery. In this retrospective study we confirmed the dependence of success of the surgery on the necessity of early treatment of the problem (up to 6 months after the appearance of initial signs), with the significance level of $P=0.037$. Any connection between age, breed or sex, and positive outcome of the surgery were not confirmed during statistical evaluation. When the horses were diagnosed early enough, an $80 \%$ success rate in surgical treatment performed within 6 months from the initial signs of crib biting was recorded, which seems to be very promising. It can be concluded that surgical treatment of crib biting by modified Forsell's myectomy is the recommended option in the therapy of this oral stereotype.
\end{abstract}

Oral stereotype, equine, surgical procedure, cribbing behaviour

The occurrence of oral stereotypes such as crib biting and aerophagy significantly influence the economic value of horses. Even though these stereotypes do not directly influence horse performance, the owners are often interested in treatment because of aesthetical reasons. In the longer time frame, other health problems can develop subsequently to this stereotype. Crib biting in horses is one of the behavioural disorders with several possibilities of neuropharmacological, mechanical or surgical therapy. Surgical therapy as one of the options is a very controversial topic in the scientific environment. Various surgical procedures have been described to treat crib biting in horses. In 1926, Forssell's procedure for the treatment of crib biting was introduced. This procedure involved segmental myectomy of the sternomandibularis, omohyoideus, and sternothyrohyoideus muscles. However, the cosmetic appearance, which is very important for many owners, was not considered. To improve the cosmetic appearance, a modified Forssell's procedure was developed (Hakanss on et al. 1992; Sarrafchi and Blokhuis 2013). Compared to the original Forssell's procedure, this modification provided less drastic changes on the horse's neck after surgery. Modified Forssell's procedure involved a bilateral neurectomy of the ventral branches of the spinal accessory nerves and partial myectomy of the omohyoideus, sternohyoideus and sternothyrohyoideus muscles (Turner et al. 1984). This method was further modified to minimize postoperative recurrence of crib biting. The new technique with more rostral transection of the sternothyrohyoideus and omohyoideus muscles was performed with a laser to reduce the risk of seroma or haematoma formation (Delacalle et al. 2002). The objective of this study was to assess the success rate of Forssell's modified myectomy in the treatment of crib biting in horses.

Address for correspondence:

Šárka Krisová

Equine Clinic

University of Veterinary and Pharmaceutical Sciences

Palackého tř. 1/3, 61242 Brno 


\section{Materials and Methods}

\section{Criteria}

This is a retrospective study of 33 horses of various breeds identified as stereotypic cribbers, which were referred to the Equine Clinic in Brno between January 2001 and December 2010. Modified Forssell's myectomy was performed on all of them. The evaluation of the results depended on the patient's database of the Equine Clinic in Brno and on information obtained from the owners by use of a standard questionnaire during the year 2012 .

\section{Retrieving Data}

Information on the recurrence of cribbing after surgical treatment was obtained by telephone conversations with the owners and trainers and evaluated after observations recorded not less than 24 months after surgery.

\section{Horses}

After follow-up data collection, 33 sport horses were involved in this study: 19 Czech warmbloods, seven Thoroughbreds, five Quarter horses, one Paint horse and one Welsh cob. There were nine stallions, eight geldings and 16 mares in the experimental group. The median age of horses was 4 years. All thoroughbreds were used for racing, warmbloods for dressage, show jumping or eventing. The Quarter horses and the Paint horse were competing in western classes and the Welsh cob was used for showing in hand.

\section{Surgical technique}

Horses under total intravenous anaesthesia were placed in dorsal recumbence and the modified Forssell's procedure was performed. To shorten the total surgery time, the surgical field was clipped and partially prepared on the standing horse.

After placing the horse in dorsal recumbency, the surgical field from rostral parts of the mandible to the caudal third of the neck, dorsolaterally to sulcus jugularis was prepared for aseptic surgery using povidone iodine solution and chlorhexidine. An incision $30 \mathrm{~cm}$ long was made on the ventral midline, starting at the level of the ramus mandibule and extended caudally. The edges of the incised skin were retracted laterally. The ventral branch of the spinal accessory nerve was located on the dorsomedial aspect of the sternomandibular muscle. Isolation was confirmed by contraction of the sternomandibular muscle after pinching the nerve with haemostat forceps. Afterwards 10-12 cm of spinal accessory nerve was removed. The same procedure was repeated on the other side of the neck. After the bilateral neurectomy, omohyoid and sternohyoid muscles were identified, released from the larynx and transected in the cranial part of the incision, then retracted caudally towards the caudal aspect of the skin incision where the omohyoid muscle was sectioned obliquely and the sternohyoid muscle transversely. Approximately $30 \mathrm{~cm}$ of muscle tissue was removed.

Subsequently, sternothyroid muscles were elevated from the trachea and resected in the full length of the skin incision. In the end, a Penrose drain was inserted into the wound and fixed at both sides of the incision. The skin was closed with a vertical mattress suture pattern, using synthetic absorbable polyfilamental suture material (Coated Vicryl 2, polyglactin 910, Ethicon). A stent bandage was applied over the incision to provide mechanical support and reduce the haematoma and seroma formation.

Potentiated sulphonamides (Norodine, Norbrook, sulphadiazine trimehtoprim, $30 \mathrm{mg} / \mathrm{kg}$ ) and non-steroidal anti-inflammatory drugs (Meflosyl, Cymedica, flunixin meglumine, $1.1 \mathrm{mg} / \mathrm{kg}$ ) were administered postoperatively. The Penrose drain was removed 4-5 days after surgery. Daily cleaning of the wound was crucial for adequate drainage and healing. In general, horses were hospitalized at the Equine Clinic in Brno for 10 days to check their overall state, comfort, feed intake, and ability to swallow. The sutures were removed on day 10 after the surgery. Patients were subsequently discharged and the owners instructed about postoperative care.

For the purposes of recording and summarizing the data, a standard questionnaire was made.

\section{Statistics}

A $\chi 2$ Chi-square test was chosen including its modifications, especially when the expected frequencies were too low (exact tests). We tested the hypothesis of independence of the chosen qualitative variable (the surgery success) on various factors (frequencies of the other qualitative variables). Differences were considered significant at $P<$ 0.05. Statistical analyses were performed using KyPlot version 2.0 software.

\section{Results}

Long-term outcome was available for 33 horses. There was no recurrence of cribbing behaviour in 20 out of 33 horses (61\%). Thirteen horses (39\%) returned to the stereotypical behaviour sooner or later after the surgery (Fig. 1). Twelve out of 20 successfully treated horses $(60 \%)$ were operated sooner than 6 months from the development of this oral stereotype (Fig. 2). On the other hand, 10 out of 13 horses, where the surgical treatment was unsuccessful, were operated more than 6 months after showing initial marks of crib 


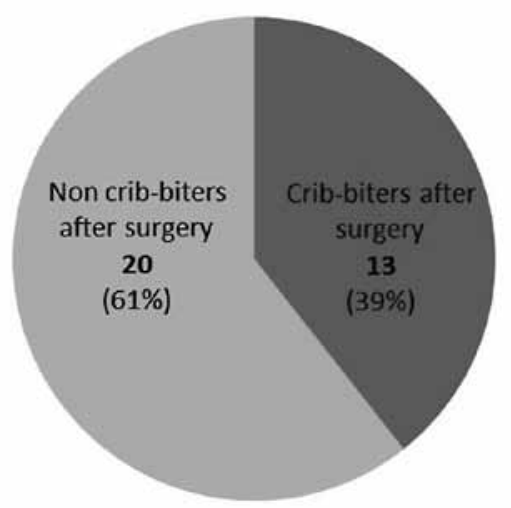

Fig. 1. Efficiency of surgery

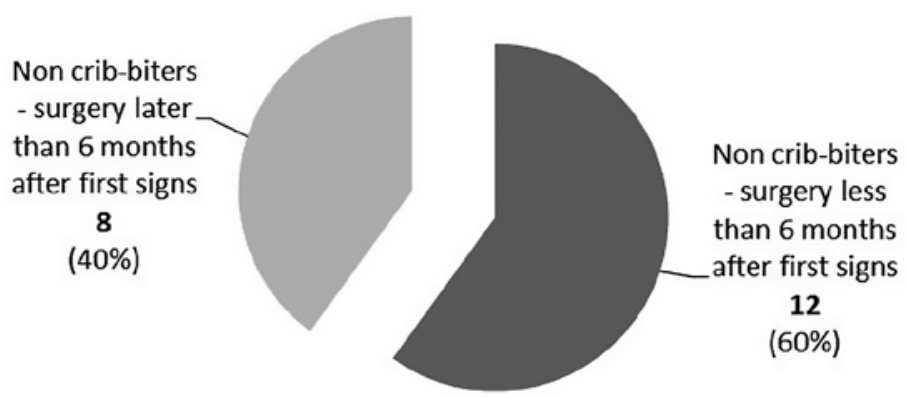

Fig. 2. Non crib-biters in the length of period of crib-biting before surgery

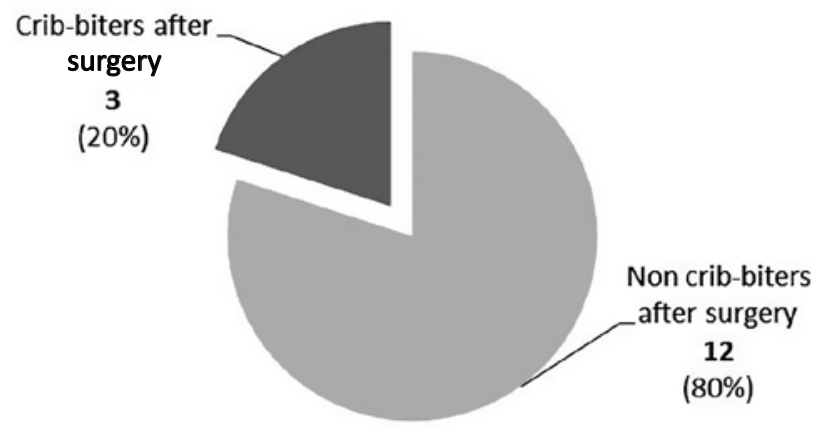

Fig. 3. Efficiency of the surgery in relation with the length of period of crib-biting before surgery - less than 6 months after first sings

biting. Eighty percent $(80 \%)$ success rate was recorded, if the surgery was performed less than 6 months after the initial signs of crib biting had been noticed (12 out of 15 horses) (Fig. 3). 
We confirmed in statistical testing the dependence of success of the surgery on the necessity of early treatment of the problem (up to 6 months after showing the initial signs), with the significance level $(P=0.037)$.

It is interesting that three out of 13 horses where the treatment was unsuccessful stopped cribbing for a certain period of time. Two of them returned to this stereotype after 6 months. One horse started crib biting again after 4 years.

There were nine stallions, eight geldings and 16 mares in the tested group. The surgery was successful in six stallions, six geldings and eight mares. Statistical analysis did not show any connection between the horse's sex and the success rate of the surgery.

Nineteen warmbloods, seven thoroughbreds, five quarter horses, one paint horse and one Welsh cob were involved. No recurrence of cribbing behaviour was confirmed in 11 warmbloods, six thoroughbreds, one quarter horse, one paint horse and one welsh cob.

Statistically, it is not possible to confirm the connection between the breed and a positive outcome of the procedure. Empirically, it seems that in our group, the best results were obtained in thoroughbreds. On the other hand, surgical treatment was least successful in quarter horses.

If we divide the patients into two groups by age, one category from 0 to 4 years and the other 5 years and more, there are 23 horses in the first category. Out of them, 15 horses were treated successfully. In the other group, 10 horses were operated on. A positive outcome was recorded in five out of these horses. Any connection between the age and success of the surgery was non-significant.

Related to the data collected in this study, change in the management after surgery was performed in 20 out of 33 horses $(61 \%)$. In 13 horses $(39 \%)$, there was no attempt to change the previous stable management. Changes in the management and feeding were taken into consideration, mostly by the owners, where the surgery was unsuccessful $(85 \%)$ - in comparison to the owners were the horses did not return to crib biting (45\%). This fact could not be statistically analysed, because the change of management was not statistically independent. It was influenced by the assumption of the success of surgery.

\section{Discussion}

In our study, modified Forssell's procedure was performed on 33 horses. A positive outcome of the surgery was confirmed in 20 horses, which means that in $61 \%$ we obtained surgical success, similar to findings reported by Turner et al. (1984) and Fjeldborg (1993). In contrast, only 30\% surgical success was reported by Schofield and Mulville (1998). Conversely, high surgical success (93\%) was mentioned in the study by Huskamp et al. (1983).

A study describing laser assisted modified Forssell's myectomy confirmed a 100\% success rate in a group of 10 horses, consisting of eight chronic crib-biters (cribbing behaviour lasted more than 2 years) and two acute crib-biters, with the stereotype lasting for 1,5 months and 4 months (Delacalle et al. 2002).

The study by Schofield and Mulville (1998), where the time aspect was mentioned, stated that complete remission was confirmed only in three out of 10 horses. Some improvement was noticed in other two horses. These five horses were crib biting from six to 18 months (median 12 months). The other five horses, where no improvement was noticed, were cribbing from six months to three years (median 18 months) (Schofield and Mulville 1998).

Results acquired by Hakans son et al. (1992) confirmed a more positive outcome of the surgery in younger horses with a shorter history of cribbing behaviour. Overall, the duration of crib-biting behaviour was longer in the horses treated unsuccessfully (Hakansson et al. 1992). 
Our results did not confirm an effect of age on the probability of a positive treatment outcome. However, we confirmed the dependency between the duration of crib biting behaviour before surgery and the probability of a successful outcome. In our group of patients, the limiting time borderline was set up to 6 months from the appearance of initial cribbing behavioural signs. This dependence was highly significant $(P<0.01)$. Especially for the horses diagnosed early enough, an $80 \%$ success rate in surgical treatment performed within 6 months from the initial signs of crib biting seems to be very promising.

Any connection between age, breed or sex and the positive outcome of the surgery was not confirmed during statistical evaluation. Although this evaluation can be influenced by the fact that the distribution of horses among these groups regarding the duration of crib biting was uneven. The duration of this habit showed to be very significant, as can be seen from the above mentioned studies.

If we wanted to divide the horses into groups according to the duration of crib biting, frequencies would be very low for statistical assessment because of the relatively small group of patients involved in this study. For example, if we created a group of mares, the majority of horses would be chronic crib-biters, thus negatively influencing the surgical treatment success rate in this sex. Similarly, two out of three unsuccessfully treated geldings or stallions were crib biting for more than 6 months.

In the category of age up to 4 years, in four horses out of eight the cribbing behaviour was not resolved. These patients were surgically treated after more than 6 months from the stereotype development. Turner et al. (1984) agree that the surgery can be considered successful if the results are evaluated at least 12 months after surgery.

In our horses, the shortest period for data collection was 2 years following surgical intervention. However, it is interesting that in one patient crib biting reappeared after 4 years. If the results were collected earlier, this treatment outcome would have been assessed as positive.

The owner stated that the horse returned to stereotypical behaviour at the moment when he was moved back to a box-stable after a previous stay on pasture. In our study, this patient was considered to be treated unsuccessfully and counted among 13 horses with a negative surgical outcome, as the purpose of the surgery was total remission of crib biting behaviour, which, in conclusion, was not accomplished. However, this case leads to the hypothesis that the positive outcome of surgery partially depends on the management and stable related factors following the surgical procedure.

In the group of Swiss horses, some breed predisposition in development of this stereotype was confirmed. An increased risk of oral stereotype appearance was observed in thoroughbred and warmblood horses (Bachmann et al. 2003). In a study from the USA, a comparison of crib biting prevalence was made among thoroughbreds, quarter horses and Arabians, confirming a higher prevalence in thoroughbred horses. The lowest appearance rate was observed in Arabian horses (Albright et al. 2009). Similar results were achieved in a study by Luescher et al. (1998), there was a significantly higher occurrence of oral stereotypes in thoroughbreds in accordance with their usage in sport and workload.

Most of the horses in our study were used for sport purposes, which is connected with many stressful situations - in training, competitions, transport, because of isolation and together with a higher risk of possible injuries and the stress of pain. Even though some genetic predisposition was confirmed in oral stereotype appearance (Vecchiotti and Galanti 1986; Kiley-Worthington 1987; Marsden 2008), it is necessary to consider breeding management and the overall usage of the horse.

The questionnaire filled out by the owners included several management related factors which could influence the occurrence of crib biting, such as the change of stabling amount, the form of daily training and feeding, and the change of the owner. Evaluation of the answers from the questionnaire confirmed that together with the surgical treatment, most 
of the owners tried to change some management factors. Most common was the change in stabling, moving the horses from the stable to pastures where presumably there was unlimited access to roughage and wider possibilities of social contact with other horses than in individual stables. Social isolation is assumed to cause a higher risk of development of oral stereotypes whereas choosing a quiet environment where the horses can express their natural behaviour reduces the stereotype occurrence rate (Kiley-Worthington 1987). The above mentioned together with an increased time for daily food intake is believed to significantly reduce the oral stereotype appearance rate (Marsden 2008). Unfortunately, many owners believe that the horses might learn this habit from their stable mates and therefore many crib biters are kept isolated, thus suffering from more stress and anxiousness (Albright et al. 2009).

Daily free movement in the paddock significantly decreases the risk of stereotypical behaviour (Bachmann et al. 2003). It was confirmed too that a daily income of roughage lower than $6.8 \mathrm{~kg}$, with hay being it is only source, increases the risk of oral stereotype development (McGreevy et al. 1995). Regular paddock access seems to be a positive factor of postoperative management, because it allows to increase daily roughage intake as well as its variability. It is suggested to offer roughage with high percentage of fibre, e.g. hay, as it reduces oral stereotypes (Kiley-Worthington 1987), most probably in two ways. Firstly, in comparison to easily digestible concentrated feeding, digestion of hay takes much longer and secondarily, high-fibre feeding can fill the stomach effectively so the horse is not motivated to search for more food (Cooper and Mason 1998).

It is apparent from the above mentioned studies that for a horse with a history of cribbing, it is recommendable to ensure unlimited access to pasture, ideally for $24 \mathrm{~h}$ a day, as protection from recurrence after surgical therapy.

The other factor, which was commonly changed following the surgery, was the usage of the horse, especially by increasing the amount of training. Some owners ( $18 \%$ of our respondents) think that the reason for the development of crib biting is boredom, so they try to make their horses busier by more intensive training. However, it has been confirmed that more intensive training does not decrease the crib biting development. In fact, the frequency increases with increased training time, particularly because the horses might be nervous and stressed after strenuous exercise (Whisher et al. 2011). This fact could influence the surgical success rate as well, especially if the change of training was too radical.

According to some authors, stable toy application reinforces development of crib biting because, in the majority of cases, toys filled with sweet treats are used (Marsden 2008). Toys seem to be ineffective as the horses spend only $1-5 \%$ of time playing with them and because the digestion of sugar potentiates the horse to develop cribbing behaviour (Whisher et al. 2011).

The overall success of the surgery without considering the influencing factors was $61 \%$. If only this percentage was taken into consideration, the success rate was sufficient, but not satisfactory enough. This retrospective study confirmed that the minimum duration of crib biting is essential for a potential positive surgery outcome. If this stereotype is treated in time, ideally up to 6 months from its first appearance, the surgery's success rate rapidly increases to $80 \%$.

We can conclude that surgical treatment of crib biting by modified Forssell's myectomy could be recommended as treatment option in the therapy of this oral stereotype.

\section{Reference}

Albright JD, Mohammed HO, Heleski CR, Wickens CL, Houpt KA 2009: Crib-biting in US horses: breed predispositions and owner perceptions of aetiology. Equine Vet J 41: 455-458

Bachmann I, Audigé L, Stauffacher M 2003: Risk factors associated with behavioral disorders of crib-biting, weaving and box-walking in Swiss horses. Equine Vet J 35: 158-163 
Cooper JJ, Mason GJ 1998: The identification of abnormal behavior and behavioural problems in stabled horses and their relationship to horse welfare: a comparative review. Equine Vet J (Suppl) 27: 5-9

Delacalle J, Burba D, Tetens J, Moore RM 2002: Nd: YAG Laser-assisted modified Forssell's procedure for treatment of cribbing (crib-biting) in horses. Vet Surg 31: 111-116

Fjeldborg J 1993: Results of surgical treatment of cribbing by neurectomy and myectomy. Equine Pract 15: 34-36

Hakansson A, Franzen P, Pettersson H 1992: Comparison of two surgical methods for treatment of crib-biting in horses. Equine Vet J 24: 494-496

Huskamp B, Henschel E, Arenhoevel H 1983: Techniques and results of a modern surgical method for preventing crib-biting. Prakt Tierarzt 64: 110-119

Kiley-sWorthington M 1987: The behaviour of horses: In relation to management and training. JA Allen, London, $265 \mathrm{p}$.

Luescher UA, McKeown DB, Dean H 1998: A cross-sectional study on compulsive behaviour (stable vices) in horses. Equine Vet J (Suppl) 27: 14-18

McGreevy PD, Nicol CJ, Cripps P, Green L, French N 1995. Management factors associated with stereotypic and redirected behaviour in the Thoroughbred horse. Equine Vet J 27: 86-91

Marsden MD 2008: Stereotypic and other behavior problems. In: Furr M and Reed S (Eds): Equine neurology. Blackwell Publishing Ltd, Oxford, UK, pp. 373-402

Schofield WL, Mulville JP 1998: Assessment of the modified Forssell's procedure for the treatment of oral stereotypies in 10 horses. Vet Rec 142: 572-575

Sarrafchi A, Blokhuis HJ 2013: Equine stereotypic behaviors: Causation, occurrence, and prevention. J Vet Behav 8: $386-394$

Turner AS, White N, Ismay J 1984: Modified Forssell's operation for crib-biting in the horse. J Am Vet Med Assoc 184: $309-312$

Vecchiotti GG, Galanti R 1986: Evidence of heredity of cribbing, weaving and stall-walking in thoroughbred horses. Livest Prod Sci 14: 91-95

Whisher L, Raum M, Pina L, Pérez L, Erb H, Houpt C, Houpt K 2011: Effects of enviromental factors on cribbing activity by horse. Appl Anim Behav Sci 135: 63-69 